\title{
Spectrum of a particle on a polyhedron enclosing a synthetic magnetic monopole
}

\author{
M.Ö. Oktel ${ }^{\mathrm{a}}$ \\ Department of Physics, Bilkent University, 06800 Ankara, Turkey
}

Received 18 August 2011

Published online 19 April 2012 - (C) EDP Sciences, Società Italiana di Fisica, Springer-Verlag 2012

\begin{abstract}
We consider a single particle hopping on a tight binding lattice formed by the vertices of a regular polyhedron and discuss the effect of a magnetic monopole enclosed in the polyhedron. The presence of the monopole induces phases on the hopping terms, given by Peierls substitution. By requiring the flux through each face of a regular polyhedron to be the same, Dirac's quantization condition is obtained in this discrete setting. For each regular polyhedron, we calculate the energy spectrum for an arbitrary value of the flux through a Dirac string coming in from one of the faces. We find that the energy levels are degenerate only when the flux through the Dirac string corresponds to a quantized monopole. We show that the degeneracies in the presence of the monopole can be classified using the double group of the symmetry of the polyhedron and label all energy levels with corresponding irreducible representations.
\end{abstract}

One of the most interesting problems in non-relativistic quantum mechanics is the study of a charged particle in the field of a magnetic monopole. As shown by Dirac [1], a consistent description of this problem requires the total magnetic flux of the monopole to be quantized in units of flux quantum $h / e$. Or, conversely, one can argue that the presence of a magnetic monopole requires electric charge to be quantized.

Beyond its implications for charge quantization and other topological arguments in field theory, the solution of Dirac's problem is used as a tool in numerical studies of the fractional quantum Hall effect. As first discussed by Haldane [2], one can imagine a particle confined to the surface of a sphere, with a magnetic monopole of strength $g$ at the center of the sphere. The energy levels of the single particle problem can be labelled by an angular momentum quantum number $l$, yielding the usual angular momentum spectrum $E=C l(l+1)$, with $C$ a constant related to the mass of the particle and the radius of the sphere. However the smallest possible value $l$ can take is $g / 2$. Thus, the lowest energy value is $g+1$ fold degenerate. One can then formally take the constant $C$ to infinity, leaving behind only the lowest degenerate manifold and hence achieve the projection the lowest Landau level. The single particle wavefunctions obtained this way forms a 'translationally invariant' basis for the numerical exact diagonalization studies.

The quantum Hall problem in the presence of a periodic potential becomes much more intricate. Even without

\footnotetext{
a e-mail: oktel@fen.bilkent.edu.tr
}

interactions, i.e. for a single particle, the energy spectrum has a complicated dependence on the magnetic field. The self-similar spectrum known as the Hofstadter butterfly displays an infinite number of gaps [3]. Furthermore, each gap in the spectrum forms a continuous region in magnetic field, and one can show that the Hall conductance within such a region is an integer which is connected to the topological properties of the phase of the wavefunction [4]. For an interacting system there is no clear picture for the interplay between the complicated single particle spectrum and interactions, more specifically the general form of fractional quantum Hall states on a lattice are not known.

While this was considered to be a purely academic problem until recently, the experimental progress on cold atoms holds promise for realization of lattice versions of the quantum Hall problem [5-9]. A lattice where magnetic field is simulated by rotation has been experimentally demonstrated [10,11], and more recently, light induced potentials have been used to create an artificial magnetic field for neutral atoms [12]. Also, theoretical investigations found that the topological properties of the gaps in the Hofstadter spectrum can be investigated within the currently achievable experimental parameters [13]. Hence, there is increased activity about the lattice studies of the fractional quantum Hall states. In recent studies, exact diagonalization has been carried out on systems of small number of particles (2-8) on small lattices with periodic boundary conditions (i.e. torus geometry) [6-8]. For small values of flux per plaquette of the lattice, one can define an effective lowest Landau level, as the width of the lowest 


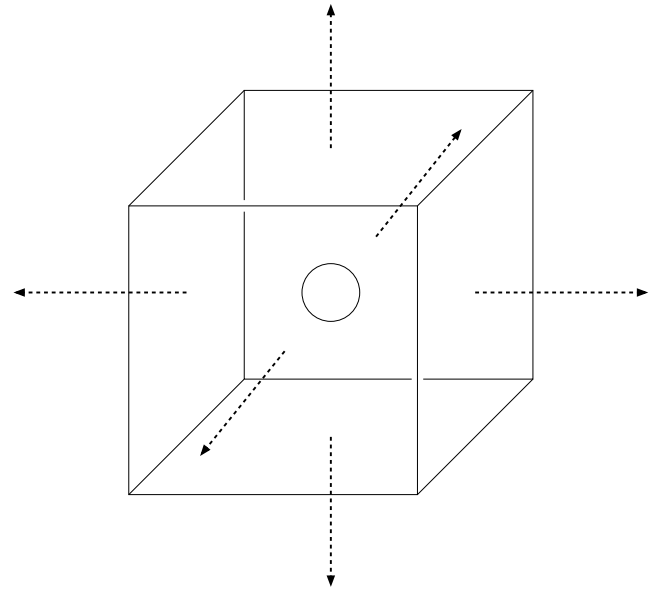

Fig. 1. A cube containing a magnetic monopole at its center. The total flux of the monopole $g \phi_{0}$ is shared by each face $\phi_{\text {face }}=g \phi_{0} / 6$.

band of the Hofstadter butterfly is much smaller compared to the gap separating it from higher states. However, near half integer flux there is no well defined lowest Landau level.

These studies naturally lead to the following question: 'would it be possible to describe the quantum mechanics of a particle moving in a closed tight binding lattice, such that each site is equivalent and the flux through each plaquette of the lattice is the same?' Equivalently, one can ask 'what is the discrete version of Dirac's magnetic monopole problem?'. In this paper, we consider this problem, calculate the energy levels for a particle on a regular polyhedron lattice enclosing a magnetic monopole and classify the degeneracies in the spectrum using group theory. While our results have implications for the discrete Quantum Hall problem outlined in the paragraph above, the system studied here can, in principle, be directly explored in an experiment using an artificial magnetic field. While the search for a monopole in the real magnetic field has so far not yielded any results, an artificial magnetic created by light coupling [12] does not have to obey Maxwell's equations and could be tailored to be a monopole field.

The paper is organized as follows: we introduce the Hamiltonian for the tight binding lattice and discussed how it is modified by the presence of the magnetic flux through plaquettes. We then consider gauge transformations in such a discrete system and recover Dirac's quantization condition for magnetic monopoles in this setting. We numerically determine the energy levels for each of the five regular polyhedra, and discuss degeneracy and periodicity properties of the system. Finally, we classify all the degenerate manifolds in the spectrum in terms of the irreducible representations of the double symmetry group of the corresponding polyhedron. The results and their bearing on the lattice quantum Hall problem are given at the end.

Let us consider tight binding cube (Fig. 1), here we assume that there is a localized state at each vertex. A particle in one of these states can make a transition to an- other state that is connected to the vertex by the edges of the cube. Thus a tight binding Hamiltonian can be written as

$$
\mathcal{H}=-t \sum_{\langle i, j\rangle} a_{i}^{\dagger} a_{j}
$$

where $t$ is the hopping strength, $a^{\dagger}, a$ are the usual creation annihilation operators for the states at the corresponding sites and the constrained $\operatorname{sum}\langle i, j\rangle$ is evaluated over pairs which are connected to each other by an edge of the polyhedron.

Now let us consider the same system, however with a magnetic monopole at the geometric center of the cube, and assume that the total magnetic flux of the monopole is $\phi_{\text {monpole }}=g \phi_{0}$. Here $\phi_{0}=h / e$ is the magnetic flux quantum and the monopole strength $g$ is not constrained yet and can be taken as a real number (Fig. 1). Due the the symmetry of the system, there will be an outward magnetic flux from each face equal to $\phi_{\text {face }}=g \phi_{0} / 6$. The Hamiltonian for the particle will be modified by the magnetic field. While this modification in the continuum is realized by minimal coupling of the vector potential to the momentum $p \rightarrow p-e A$, in the discrete tight binding model corresponding modification is achieved by giving phases to hopping terms in the Hamiltonian. This method, known as the 'Peierls substitution' can be justified within the tight binding approximation [3,14-17], and as a result correctly describes the phase gained by the particle upon traversing an orbit that encloses certain amount of magnetic flux. In our case the modified Hamiltonian can be written as

$$
\mathcal{H}_{\text {mag }}=-t \sum_{\langle i, j\rangle} e^{i 2 \pi A_{i j}} a_{i}^{\dagger} a_{j} .
$$

Here the hopping phases $A_{i j}=-A_{j i}$ are chosen so that their sum over a closed path gives the total magnetic flux through the surface bounded by that closed path. In particular for each face

$$
\sum_{\text {face }} A_{i j}=\phi_{\text {face }} / \phi_{0}
$$

Here the sign of the flux is related to the direction of the sum by the right hand rule. There is certain freedom in the choice of the link variables $A_{i j}$, the only requirement being that they satisfy the above condition. This is the reflection of the gauge freedom in the continuous problem to the lattice problem.

Once a certain selection for the gauge, and hence $A_{i j}$ are made and the Hamiltonian is written in the form equation (2), one can immediately notice that there is a difficulty in the description of any system that is enclosing a monopole. When the total flux leaving the surface of the polyhedron is calculated

$$
\Phi_{\text {total }}=\sum_{\text {all faces }} \phi_{\text {face }}=\phi_{0} \sum_{\text {all faces }} \sum_{\text {face }} A_{i j}=0,
$$

it is found to be zero as each link is traversed twice, once in each direction. So it would seem that no choice of $A_{i j}$ would be sufficient to describe the enclosed monopole. 


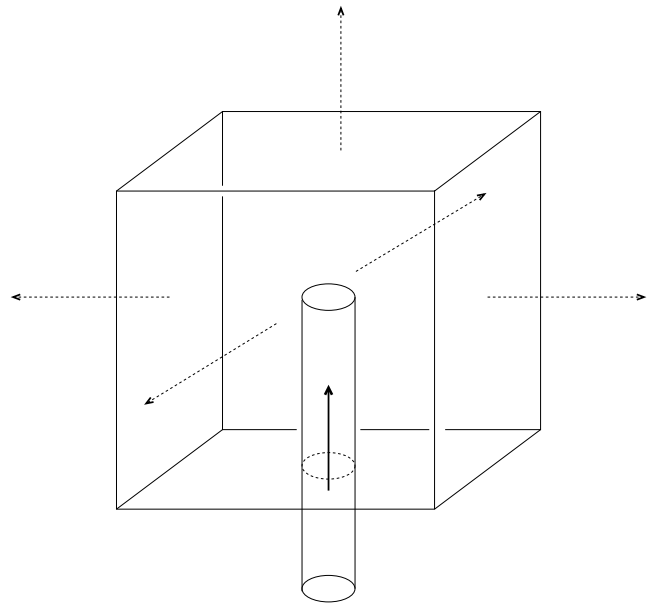

Fig. 2. A cube containing a Dirac string bringing in the magnetic flux through the bottom face. All the faces except the bottom have flux $\phi_{\text {face }}=g \phi_{0} / 6$ while the bottom face has $\phi_{\text {face }}=-5 g \phi_{0} / 6$.

Once again making the correspondence to the continuum problem, this realization is no different from the realization that the field of a monopole can not be described by a single valued non-singular vector potential on the sphere. Not surprisingly, the resolution follows the same lines.

The sum of the hopping phases $A_{i j}$ around a face essentially describes the total phase the particle accumulates when it goes once around the face. This quantity, being a phase, is defined up to $2 \pi$. In other words selection of different $A_{i j}$ which will increase this phase by an integer multiple of $2 \pi$ would just correspond to a gauge transformation. Thus, one can imagine describing the monopole through the following selection of $A_{i j}$. First choose $A_{i j}$ so that the flux through each face but one is equal to $\phi_{\text {face }}$. Now because the sum of the flux through all faces is equal to zero, flux through the remaining face would be, $-(N-1) \phi_{\text {face }}$ where $N$ is the number of faces of the polyhedron. Now this flux would generate a phase $-(N-1) \phi_{\text {face }} / \phi_{0}$. If this phase can be related to the phase on the other faces by adding an integer multiple of $2 \pi$, then one can choose an alternative gauge which defines the flux through this face to be $\phi_{\text {face }}$.

Thus to be able to describe the magnetic monopole consistently we require

$$
-(N-1) \phi_{\text {face }} / \phi_{0}=\phi_{\text {face }} / \phi_{0}-m 2 \pi,
$$

with $m$ an integer. Thus we get

$$
N \phi_{\text {face }} / \phi_{0}=\phi_{\text {monopole }} / \phi_{0}=g=m \text {, }
$$

consequently, $g$ has to be an integer. Hence, we recover the the Dirac quantization condition that the total magnetic flux emitted by a magnetic monopole has to be an integer multiple of the flux quantum.

The above argument can be repeated much more clearly if one thinks about bringing the flux to the monopole by using an infinite solenoid, i.e., a Dirac string (Fig. 2). The description of such a string in the present

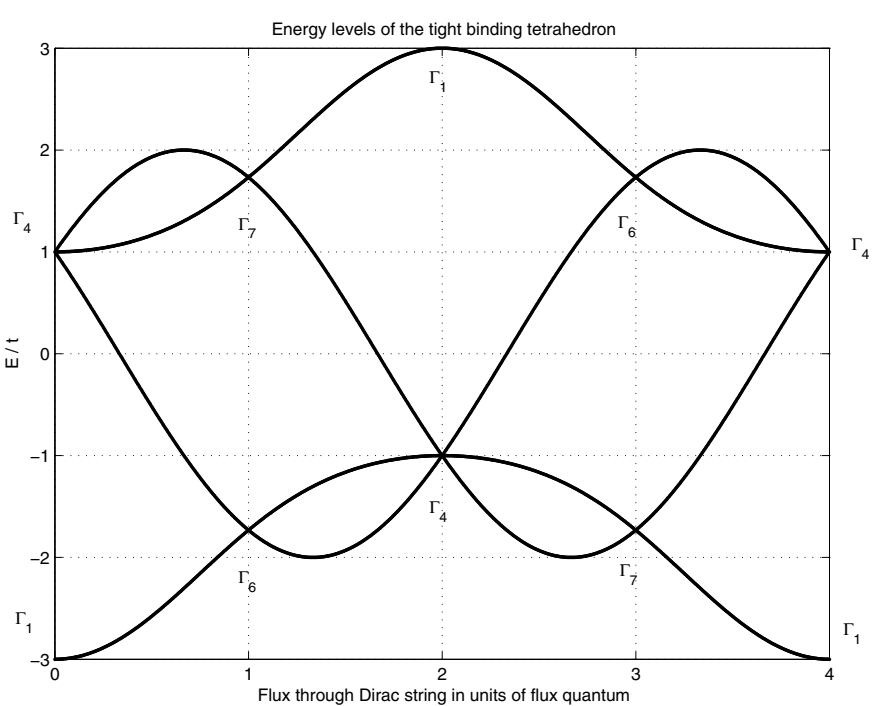

Fig. 3. Energy levels of the tight binding tetrahedron as a function of the flux through the Dirac String. The degeneracies at integer values of the flux, corresponding to monopoles are labelled by irreducible representations of the double tetrahedral group Table 1.

discrete case is much simpler compared to continuum as the string can be brought into the polyhedron without crossing the path of the particle. One can calculate the spectra for an arbitrary value of the flux $g \phi_{0}$ through the Dirac string. However it is only at the integer values of $g$ the Dirac string actually describes a magnetic monopole, as the string can be made to 'disappear' by a gauge transformation.

We numerically calculated the energy spectrum for an arbitrary value of $g$, for all five platonic solids (tetrahedron, octahedron, cube, icosahedron and dodecahedron). This calculation is carried out for values $0 \leq g \leq N$ where $N$ is the number of faces of the polyhedron, as the spectrum is periodic with the period $N$. Adding $N$ units of flux quantum to the Dirac string would correspond to increasing the phase around each loop by $2 \pi$. Our results are plotted in Figures 3-7, where one can observe that the degeneracies in the spectra occur only at integer values of $g$, exactly at the values that correspond to magnetic monopoles. The reason for these degeneracies is clearly the restoration of the symmetry of the polyhedron, when the flux through each of its faces is equal. The rest of the paper is focused on an investigation of these degeneracies.

Each of the platonic solids posses operations that leave the polyhedron invariant. These symmetry operations form a group within themselves, and furthermore they all commute with the Hamiltonian describing the tight binding dynamics of the particle. Hence, the degeneracies of the spectra can be labelled by the irreducible representations of the corresponding group. This labelling can be obtained straightforwardly by explicitly constructing the a few of the symmetry operations from different classes of the symmetry group and comparing their characters with the character table of the group.

For example, consider the eigenvalues found for the tetrahedron at zero field. The characters for one $C_{3}$ 


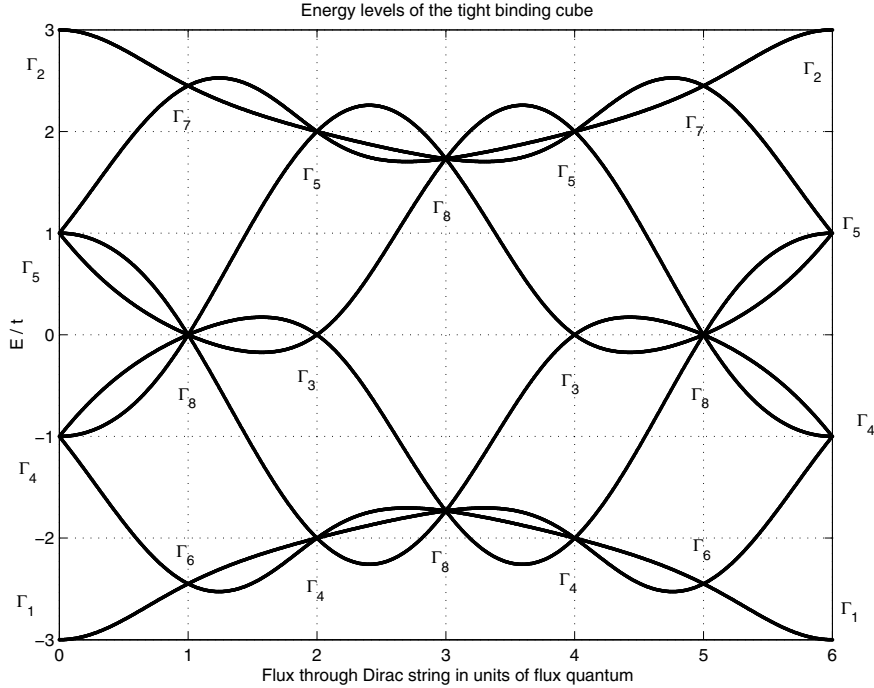

Fig. 4. Energy levels of the tight binding cube as a function of the flux through the Dirac String. The degeneracies at integer values of the flux, corresponding to monopoles are labelled by irreducible representations of the double octahedral group Table 2. The cube is the only bipartite polytope, hence the energy spectrum has $E \rightarrow-E$ symmetry.

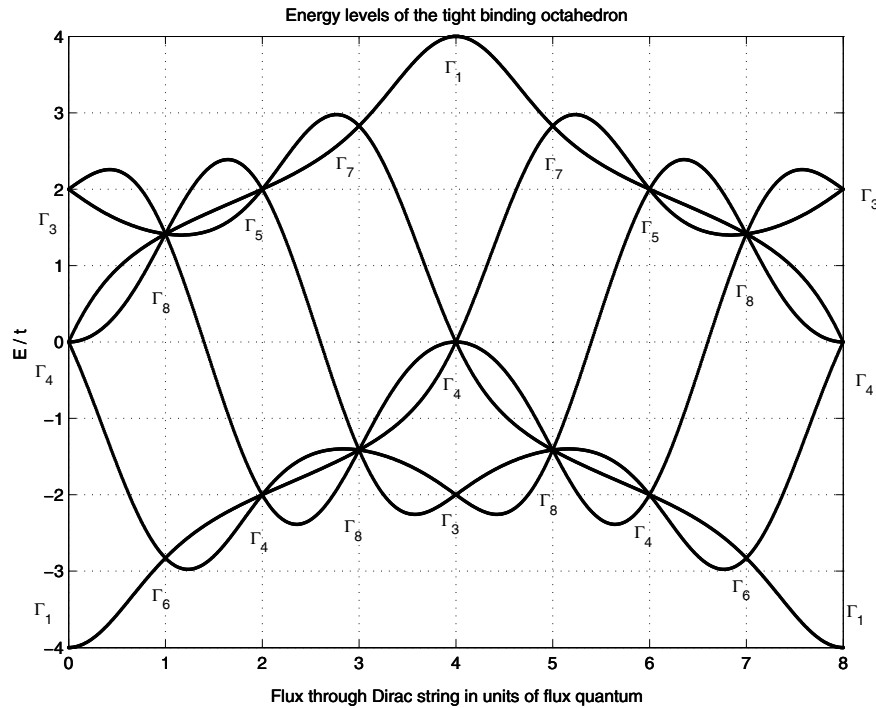

Fig. 5. Energy levels of the tight binding octahedron as a function of the flux through the Dirac String. The degeneracies at integer values of the flux, corresponding to monopoles are labelled by irreducible representations of the double octahedral group Table 2 .

rotation around any of the axis reveals that the the nondegenerate ground state is related to the irreducible representation $\Gamma_{1}$, from Table 1 . Similarly the three fold degeneracy of the excited states form the basis for the $\Gamma_{4}$ irreducible representation of the group.

In the presence of the magnetic monopole, however, the situation is more complicated. While all the elements of the group still commute with the Hamiltonian, the multiplication of two elements in the group give the corresponding element in the multiplication table of the group

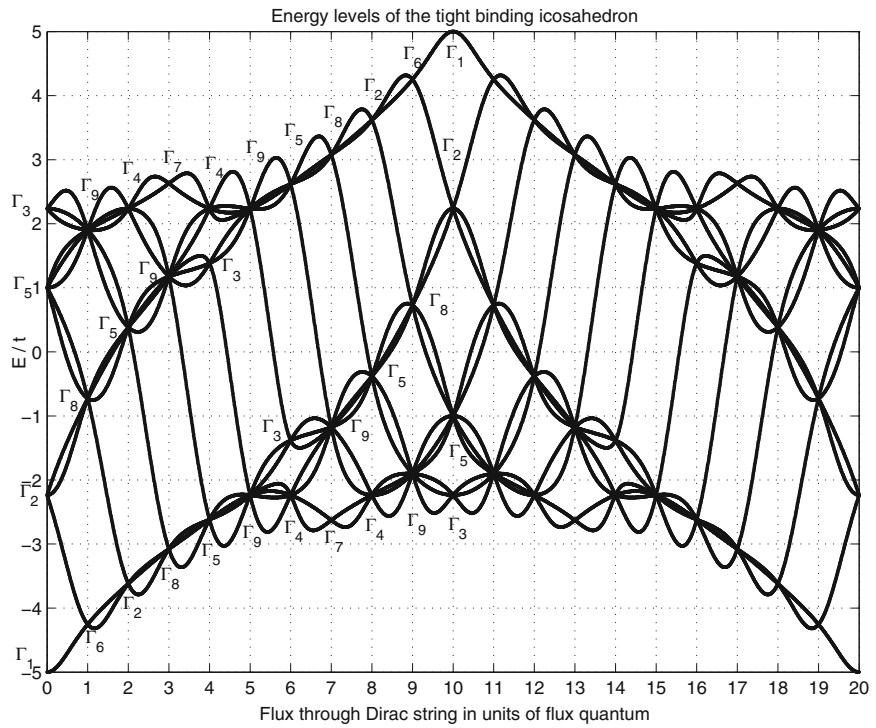

Fig. 6. Energy levels of the tight binding icosahedron as a function of the flux through the Dirac String. The degeneracies at integer values of the flux, corresponding to monopoles are labelled by irreducible representations of the double icosahedral group Table 3 .

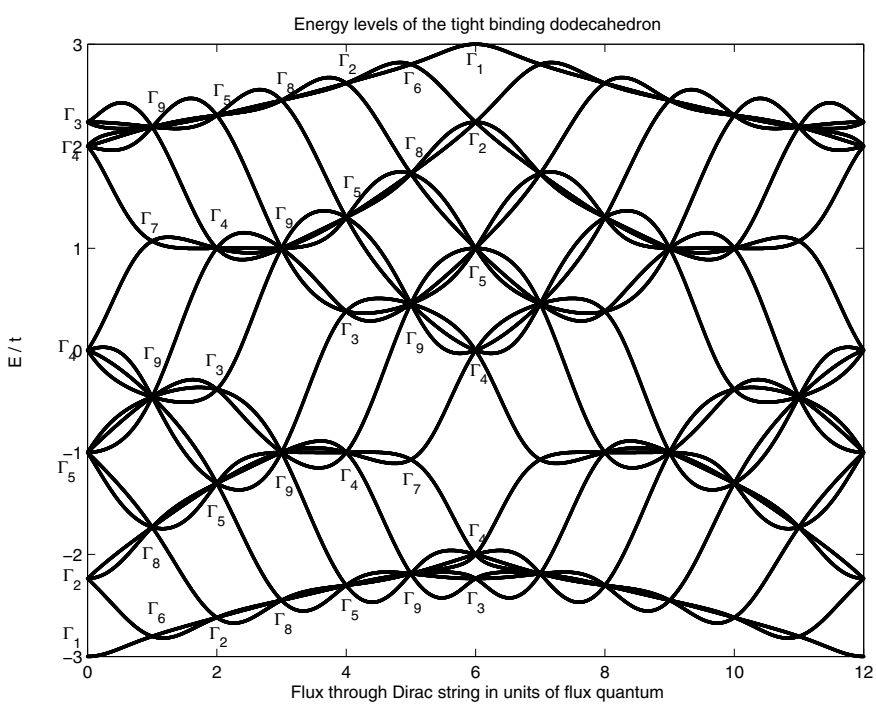

Fig. 7. Energy levels of the tight binding dodecahedron as a function of the flux through the Dirac String. The degeneracies at integer values of the flux, corresponding to monopoles are labelled by irreducible representations of the double icosahedral group Table 3.

only up to a factor. The new symmetry operations which commute with the Hamiltonian are not pure rotations, but involve rotations plus a gauge transformation. These operations are similar to the operations of the Magnetic translation group $[18,19]$, defined for a two dimensional periodic potential in a perpendicular magnetic field. Essentially, one can observe that the action of symmetry operations any degenerate manifold of the Hamiltonian define matrices which form a ray (or projective) representation of the symmetry group of the Hamiltonian. 
Table 1. The character table for the double tetrahedral group. $\omega=\exp i \frac{2 \pi}{3}$.

\begin{tabular}{cccccccc}
\hline$T^{\prime}$ & $E$ & $R$ & $6 C_{2}$ & $4 C_{3}$ & $4 C_{3}^{\prime}$ & $4 C_{3} R$ & $4 C_{3} R^{\prime}$ \\
\hline$\Gamma_{1}$ & 1 & 1 & 1 & 1 & 1 & 1 & 1 \\
$\Gamma_{2}$ & 1 & 1 & 1 & $\omega$ & $\omega$ & $\omega^{2}$ & $\omega^{2}$ \\
$\Gamma_{3}$ & 1 & 1 & 1 & $\omega^{2}$ & $\omega^{2}$ & $\omega$ & $\omega$ \\
$\Gamma_{4}$ & 3 & 3 & -1 & 0 & 0 & 0 & 0 \\
\hline$\Gamma_{5}$ & 2 & -2 & 0 & 1 & -1 & 1 & -1 \\
$\Gamma_{6}$ & 2 & -2 & 0 & $\omega$ & $-\omega$ & $\omega^{2}$ & $-\omega^{2}$ \\
$\Gamma_{7}$ & 2 & -2 & 0 & $\omega^{2}$ & $-\omega^{2}$ & $\omega$ & $-\omega$ \\
\hline
\end{tabular}

Table 2. The character table for the double octahedral group.

\begin{tabular}{ccccccccc}
\hline$O^{\prime}$ & $E$ & $R$ & $6 C_{2}$ & $6 C_{4}$ & $6 C_{4}^{\prime}$ & $12 C_{2} R$ & $8 C_{3}$ & $8 C_{3}^{\prime}$ \\
\hline$\Gamma_{1}$ & 1 & 1 & 1 & 1 & 1 & 1 & 1 & 1 \\
$\Gamma_{2}$ & 1 & 1 & 1 & -1 & -1 & -1 & 1 & 1 \\
$\Gamma_{3}$ & 2 & 2 & 2 & 0 & 0 & 0 & -1 & -1 \\
$\Gamma_{4}$ & 3 & 3 & -1 & 1 & 1 & -1 & 0 & 0 \\
$\Gamma_{5}$ & 3 & 3 & -1 & -1 & -1 & 1 & 0 & 0 \\
\hline$\Gamma_{6}$ & 2 & -2 & 0 & $\sqrt{2}$ & $-\sqrt{2}$ & 0 & 1 & -1 \\
$\Gamma_{7}$ & 2 & -2 & 0 & $-\sqrt{2}$ & $\sqrt{2}$ & 0 & 1 & -1 \\
$\Gamma_{8}$ & 4 & -4 & 0 & 0 & 0 & 0 & -1 & 1 \\
\hline
\end{tabular}

Table 3. The character table for the double icosahedral group. $\alpha=(1+\sqrt{5}) / 2, \beta=(1-\sqrt{5}) / 2$.

\begin{tabular}{cccccccccc}
\hline$I^{\prime}$ & $E$ & $R$ & $12 C_{5}$ & $12 C_{5}^{\prime}$ & $12 C_{5} R$ & $12 C_{5} R^{\prime}$ & $20 C_{3}$ & $20 C_{3}^{\prime}$ & $30 C_{2}$ \\
\hline$\Gamma_{1}$ & 1 & 1 & 1 & 1 & 1 & 1 & 1 & 1 & 1 \\
$\Gamma_{2}$ & 3 & 3 & $\alpha$ & $\alpha$ & $\beta$ & $\beta$ & 0 & 0 & -1 \\
$\Gamma_{3}$ & 3 & 3 & $\beta$ & $\beta$ & $\alpha$ & $\alpha$ & 0 & 0 & -1 \\
$\Gamma_{4}$ & 4 & 4 & -1 & -1 & -1 & -1 & 1 & 1 & 0 \\
$\Gamma_{5}$ & 5 & 5 & 0 & 0 & 0 & 0 & -1 & -1 & 1 \\
\hline$\Gamma_{6}$ & 2 & -2 & $\alpha$ & $-\alpha$ & $-\beta$ & $\beta$ & 1 & -1 & 0 \\
$\Gamma_{7}$ & 2 & -2 & $\beta$ & $-\beta$ & $-\alpha$ & $\alpha$ & 1 & -1 & 0 \\
$\Gamma_{8}$ & 4 & -4 & 1 & -1 & -1 & 1 & -1 & 1 & 0 \\
$\Gamma_{9}$ & 6 & -6 & -1 & 1 & 1 & -1 & 0 & 0 & 0 \\
\hline
\end{tabular}

In other words, if we have two elements of the symmetry group $p_{1}$ and $p_{2}$, which upon group multiplication give $p_{3}$, in the zero magnetic field case we get a representation in terms of the matrices:

$$
D\left(p_{1}\right) D\left(p_{2}\right)=D\left(p_{3}\right)
$$

However with the magnetic monopole present, this multiplication is modified by a phase, i.e., the corresponding representation under the magnetic field satisfies

$$
D^{\prime}\left(p_{1}\right) D^{\prime}\left(p_{2}\right)=u\left(p_{1}, p_{2}, p_{3}\right) D^{\prime}\left(p_{3}\right),
$$

where $u$ is a complex number, which is one of the $h$ roots of unity, with $h$ the order of the group. The projective representations of point groups have been studied by Janssen [20] and also by Boyle and Green [21]. However in the present case it turns out that enumeration and detailed study of the projective representations is not necessary. All the group elements in the tetrahedral, octahedral and icosahedral group are rotations, and they form a sub-
Table 4. The ground state irreducible representations and corresponding degeneracies of the five regular polyhedra.

\begin{tabular}{cccccc}
\hline Polyhedron faces & 4 & 6 & 8 & 12 & 20 \\
\hline$\phi=0$ & $\Gamma_{1}(1)$ & $\Gamma_{1}(1)$ & $\Gamma_{1}(1)$ & $\Gamma_{1}(1)$ & $\Gamma_{1}(1)$ \\
$\phi=1$ & $\Gamma_{6}(2)$ & $\Gamma_{6}(2)$ & $\Gamma_{6}(2)$ & $\Gamma_{6}(2)$ & $\Gamma_{6}(2)$ \\
$\phi=2$ & $\Gamma_{4}(3)$ & $\Gamma_{4}(3)$ & $\Gamma_{4}(3)$ & $\Gamma_{2}(3)$ & $\Gamma_{2}(3)$ \\
$\phi=3$ & & $\Gamma_{8}(4)$ & $\Gamma_{8}(4)$ & $\Gamma_{8}(4)$ & $\Gamma_{8}(4)$ \\
$\phi=4$ & & & $\Gamma_{3}(2)$ & $\Gamma_{5}(5)$ & $\Gamma_{5}(5)$ \\
$\phi=5$ & & & & $\Gamma_{9}(6)$ & $\Gamma_{9}(6)$ \\
$\phi=6$ & & & & $\Gamma_{3}(3)$ & $\Gamma_{4}(4)$ \\
$\phi=7$ & & & & & $\Gamma_{7}(2)$ \\
$\phi=8$ & & & & & $\Gamma_{4}(4)$ \\
$\phi=9$ & & & & & $\Gamma_{9}(6)$ \\
$\phi=10$ & & & & & $\Gamma_{3}(3)$ \\
\hline
\end{tabular}

group of the continuous group $\mathrm{O}(3)$. One can use the double isomorphism between $\mathrm{O}(3)$, and $\mathrm{SU}(2)$, to extend the finite subgroups to double groups by introducing a group operation $R$, which corresponds to rotation by $2 \pi[22]$. Once the character tables for the double groups are constructed, energy spectrum for the polyhedra containing magnetic monopoles can be entirely labelled in terms of the irreducible representation of the double groups. As in the continuum case, if the total magnetic flux from the monopole is even, the energy eigenstates will be labelled only in terms of the original irreducible representations, while for odd magnetic flux the labelling will be in terms of the irreducible representations that appear as a result of the doubling of the group.

For the tetrahedron, we give the character table of the double tetrahedral group in Table 1, for the cube and the octahedron the relevant table is that of the double octahedral group Table 2. Finally, the degeneracies of the icosahedron and the dodecahedron are labelled using the character table of the double icosahedral group Table 3. We did not consider the groups to include inversions, as the inclusion of inversions are not needed to resolve any further degeneracies.

For all the Platonic solids, we list the ground state degeneracy and the corresponding irreducible representation is given in Table 4. One must notice that the ground state degeneracy for monopole strength $g$ is not always equal to $g+1$ as in continuum. For example the octahedron containing a 4 flux quanta has a twofold degenerate ground state as opposed to the expected value 5 . The evolution of the states for continuous $g$, as plotted in the figures, suggest that in all such cases on can view the degeneracy of the ground state to be lifted through the reduction of the symmetry group from the full spherical symmetry. Thus the splitting is in a sense similar to the splitting of the Landau levels by the application of a periodic potential, forming the well known Hofstadter spectrum.

In conclusion, we have presented the Dirac quantization condition in a discrete setting and argued how monopoles can be introduced to a tight binding lattice calculation. Using this general framework we have calculated the energy levels of the lattices formed by the vertices 
of five platonic solids, and then classified all the degeneracies with respect to the double symmetry group of the solid. This classification, given in Figures 3-7, and Table 4 forms the main result of this paper. The irreducible representations of the ground state manifold will be useful for numerical calculations of many body states on these lattices as it can cut down dimension of the many-body Hilbert space dramatically. As the current studies of fractional quantum Hall states are limited to a very small (28) particles, the limited number of states on the regular polyhedra here do not pose a significant constraint. Projection of single particle wavefunctions into a particular irreducible representation can be used to look for lattice quantum Hall states with larger number of particles.

We would like to thank Huzeyfe Yilmaz, Seyda Ipek, Ahmet Keles, Onur Umucalilar and particularly Tekin Dereli for useful discussions. M.O.O. is supported by a TUBA-GEBIP grant, and TUBITAK grant No. 109T267.

\section{References}

1. P.A.M. Dirac, Proc. R. Soc. Lond. 133, 60 (1931)

2. F.D.M. Haldane, Phys. Rev. Lett. 51, 605 (1983)

3. D.R. Hofstadter, Phys. Rev. B 14, 2239 (1976)

4. D.J. Thouless, M. Kohmoto, M.P. Nightingale, M. den Nijs, Phys. Rev. Lett. 49, 405 (1982)

5. A.S. Sørensen, E. Demler, M.D. Lukin, Phys. Rev. Lett. 94, $086803(2005)$
6. M. Hafezi, A.S. Sørensen, E. Demler, M.D. Lukink, Phys. Rev. A 76, 023613 (2007)

7. D. Jaksch, P. Zoller, New J. Phys. 5, 56 (2003)

8. R.N. Palmer, D. Jaksch, Phys. Rev. Lett. 96, 180407 (2006)

9. R.O. Umucalılar, M.Ö. Oktel, Phys. Rev. A 76, 055601 (2007)

10. S. Tung, V. Schweikhard, E.A. Cornell, Phys. Rev. Lett. 97, 240402 (2006)

11. R.A. Williams, S. Al-Assam, C.J. Foot, Phys. Rev. Lett. 104, 050404 (2010)

12. Y.-J. Lin, R.L. Compton, K. Jiménez-García, J.V. Porto, I.B. Spielman, Nature 462, 628 (2009)

13. R.O. Umucalllar, H. Zhai, M.Ö. Oktel, Phys. Rev. Lett. 100, 070402 (2008)

14. R. Peierls, Z. Phys. 80, 763 (1933)

15. G.M. Obermair, H.-J. Schellnhuber, Phys. Rev. B 23, 5185 (1981)

16. H.-J. Schellnhuber, G.M. Obermair, A. Rauh, Phys. Rev. B 23, 5191 (1981)

17. R.O. Umucalilar, M.Ö. Oktel, Phys. Rev. A 78, 033602 (2008)

18. J. Zak, Phys. Rev. 134, A1602 (1964)

19. E. Brown, Phys. Rev. 133, A1038 (1964)

20. T. Janssen, Crystallographic groups (North Holland, Amsterdam, 1974)

21. L.L. Boyle, K.F. Green, Phil. Trans. R. Soc. Lond. 288, 237 (1978)

22. H. Weyl, The theory of groups and quantum mechanics (Methuen, London, 1931) 